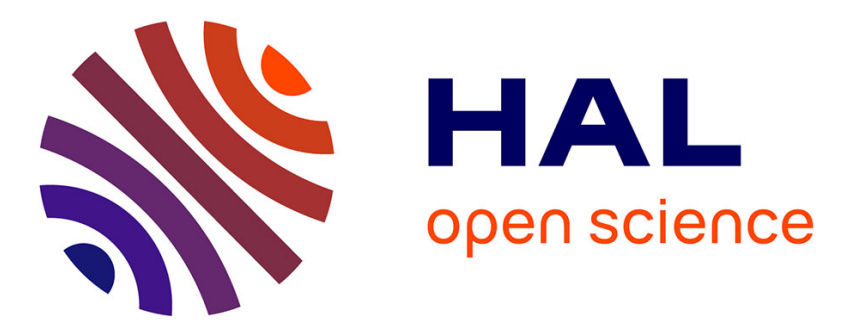

\title{
Conformational anisotropy of liquid crystalline side chain polymers: a small angle neutron scattering study
}

F. Moussa, J.P. Cotton, F. Hardouin, P. Keller, M. Lambert, G. Pépy, Monique Mauzac, H. Richard

\section{- To cite this version:}

F. Moussa, J.P. Cotton, F. Hardouin, P. Keller, M. Lambert, et al.. Conformational anisotropy of liquid crystalline side chain polymers: a small angle neutron scattering study. Journal de Physique, 1987, 48 (7), pp.1079-1083. 10.1051/jphys:019870048070107900 • jpa-00210529

\section{HAL Id: jpa-00210529 \\ https://hal.science/jpa-00210529}

Submitted on 1 Jan 1987

HAL is a multi-disciplinary open access archive for the deposit and dissemination of scientific research documents, whether they are published or not. The documents may come from teaching and research institutions in France or abroad, or from public or private research centers.
L'archive ouverte pluridisciplinaire HAL, est destinée au dépôt et à la diffusion de documents scientifiques de niveau recherche, publiés ou non, émanant des établissements d'enseignement et de recherche français ou étrangers, des laboratoires publics ou privés. 


\title{
LE JOURNAL DE PHYSIQUE
}

\section{CONFORMATIONAL ANISOTROPY OF LIQUID CRYSTALLINE SIDE CHAIN POLYMERS : A SMALL ANGLE NEUTRON SCATTERING STUDY}

\author{
F. Moussa, J.P. Cotton, F. Hardouin, ${ }^{*}$ P. Keller, M. Lambert, G. Pépy, M. Mauzac ${ }^{+}$and H. Richard ${ }^{+}$ \\ Laboratoire Commun CEA-CNRS Léon Brillouin, CEN Saclay, 91191 Gif-sur-Yvette Cedex, France \\ ${ }^{+}$Centre de Recherche Paul Pascal, Université de Bordeaux I, 33405 Talence Cedex, France
}

(Reçu le 7 mai 1987, accepté le 19 mai 1987)

\begin{abstract}
Résumé.-On a mesuré, au moyen de la diffusion de neutrons aux petits angles, l'anisotropie de conformation de deux types de polymères cristaux liquides en peigne : un polyméthacrylate et un polyméthylsiloxane, dans leur phase nématique et smectique. On a déterminé les tailles caractéristiques (parallèle et perpendiculaire au directeur) d'une chaîne marquée et leur évolution avec la température
\end{abstract}

\begin{abstract}
The anisotropy of the conformation of two kinds of comblike liquid crystal polymers, a polymethacrylate and a polymethylsiloxane has been measured in the nematic and smectic phases by small angle neutron scattering (SANS). The global sizes (parallel and perpendicular to the director) of one labelled chain and their temperature dependence have been determined.
\end{abstract}

In recent years the liquid crystal (LC) polymers have given rise to a growing interest in both theoretical and experimental physics [1]. In terms of competition between mesophase and random coil-formation, a main question raised is the influence on the backbone of the polymer of an anisotropic nematic or smectic field induced by its hanging mesogenic moieties. Only a few experimental results exist in this domain [2-5]. Theoretical predictions concerning the polymer conformation and its thermal evolution appeared recently $[6,7]$. In a previous paper [4] we performed a small angle neutron scattering (SANS) study on a LC side chain polymethacrylate. The labelling technique (deuteration) allowed us to look at the conformation of one chain among the others. These results showed that the backbone exhibited little anisotropy in the nematic phase and much more in the smectic $A$ phase where, at the

* Permanent address : Centre de Recherche Paul Pascal, Univ. Bordeaux I, 33405 Talence Cedex, France. temperature of experiment, it was confined to one or two layers. From neutron diffraction (ND) measurements we concluded that a local bilayered order was present with a tendency to a pseudo periodicity of the backbone in one or two smectic layers.

It is interesting to extend this earlier work with a study of the effect of replacing the alkoxy tail $\mathrm{OC}_{4} \mathrm{H}_{9}$ of this polymethacrylate by a polar end-group $\mathrm{CN}$ and to measure the temperature dependence of the backboneanisotropy. We report also results concerning another polymer : a side chain polymethyl-siloxane.

The samples are mixtures of liquid crystal polymers hydrogenated with partially deuterated ones. Two different compounds have been studied :

(1) The polymethacrylates with a CN-tail (PMACN) were synthetized at L.L.B. [4]. The molecular weight is much lower than 300.000 (see the values of the radius of gyration given subsequently) but needs to be defined more precisely. 
The PMA-CN partially deuterated polymer has the form :

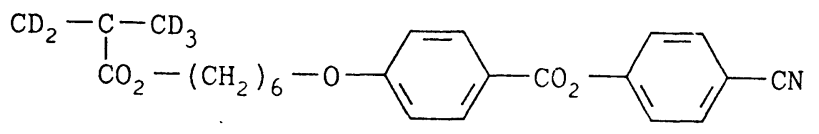

A differential scanning calorimetry (DSC) diagram allows us to specify the sequence of mesophase transition temperatures (Tab. I).

(2) The substituted polymethylsiloxanes (PMS) have been synthetized at C.R.P.P.[8] with a molecular weight $\simeq 15000$ and a polydispersity of 1.2 . Let us notice that here the deuteration consists of replacing the tail $-\mathrm{OCH}_{3}$ by $-\mathrm{OCD}_{3}$.

The PMS partially deuterated polymer has the form :

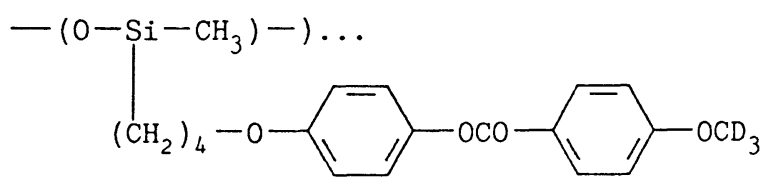

The SANS experiments were obtained with the spectrometer PAXY at Orphée Reactor. An $X Y$ position sensitive detector with cell size $5 \times 5 \mathrm{~mm}^{2}$ located at $2 \mathrm{~m}$ from the sample was essential in measuring the anisotropic intensity distributions. Two wavelengths $\lambda$ were used $\lambda=15.1 \AA$ for SANS experiments covering the scattering vector range $8 \times 10^{-3} \leq q \leq$ $3 \times 10^{-2} \AA^{-1}$, and $\lambda=2.97 \AA$ for ND experiments $\left(0.04 \leq q \leq 0.5 \AA^{-1}\right)$. The large wavelength distribution used $(\Delta \lambda / \lambda \simeq 20 \%)$ is easily taken into account [9] in measurements of radii of gyration.

The samples (thickness $1 \mathrm{~mm}, 15 \mathrm{~mm}$ of diameter, neutron beam diameter $7 \mathrm{~mm}$ ) were set in an oven (temperature regulation better than $0.2^{\circ} \mathrm{C}$ ). The oven was then placed in a magnetic field of $1.4 T$ in order to obtain aligned nematic and smectic phases. is always observed corresponding to a layer spacing of $35 \AA$ already measured by X-ray experiments [2]. It is much greater than the length of hanging moieties thus, the smectic A layers of PMA-CN are made from mesogenic groups which partially overlap as usually observed for terminally polar smectogens (i.e. partially bilayered $\mathrm{S}_{\mathrm{A}}$ phase of low molecular weight compounds). As for PMA $-\mathrm{C}_{4} \mathrm{H}_{9}$, no second order peak in diffraction is observed on smectic layers but third order peak is present. The deuteration does not yield additional diffuse spots so the diffraction spectra of PMA-CN are less rich than the diffraction measurements on PMA $-\mathrm{C}_{4} \mathrm{H}_{9}$ with a deuterated backbone. The latter [4] exhibits diffuse spots which are proof of a local bilayered order connected to a pseudo-periodicity of the backbone, whereas the long range order is that of a monolayered smectic $A\left(S_{A}\right)$. The anisotropy of the backbone is measured by SANS. If $q / /$ and $q_{\perp}$ are the components of $q$ parallel and perpendicular to the magnetic field which alignes the liquid crystal medium, then the corresponding scattered intensities are given by :

$$
\begin{aligned}
I^{-1}\left(q_{/ /}\right) & =I^{-1}(0)\left[1+q_{/ /}^{2} R_{/ /}^{2}\right] ; q_{/ /} \cdot R_{/ /} \leqslant 1 \\
I^{-1}\left(q_{\perp}\right) & =I^{-1}(0)\left[1+q_{\perp}^{2} R_{\perp}^{2}\right] ; q_{\perp} \cdot R_{\perp} \leqslant 1
\end{aligned}
$$

$R_{/ /}$and $R_{\perp}$ are the quadratic characteristic sizes of the polymer, respectively parallel and perpendicular to the nematic director. Their temperature dependent values are shown in figure 1 . The backbone has always an anisotropic conformation but much less than in the case of $\mathrm{PMA}-\mathrm{C}_{4} \mathrm{H}_{9}$ (in this compound $R_{\perp} / R_{/ /} \approx 4$ for the temperature of measurement). It is always more or less confined in one smectic layer but its extension perpendicularly to the magnetic field is only 1.4 times greater than its extension along the magnetic field. Several parameters may determine the conformation such as a very much smaller molecular weight for PMA-CN and the lack of separate sublayers in its

Table I.-Transition temperatures of PMA-CN and PMS

\begin{tabular}{|c|cccc|}
\hline Polymer & \multicolumn{4}{|c|}{ Transition Temperature $\left({ }^{\circ} \mathrm{C}\right)$} \\
\hline PMA - CN & Glassy 50 & Smectic 92 & Nematic 108 & Liquid \\
PMS - $\mathrm{OCH}_{3}$ & Glassy 7 & Smectic 74 & Nematic 104 & Liquid \\
\hline
\end{tabular}

The aligned liquid crystal phases of the sample were obtained in the way described in (4). The degree of alignment was measured from the mosaicity of 001 Bragg peak, it was less than $5^{\circ}$. Since we have already verified once that the sizes deduced from SANS were independent of the concentration of labelled molecules [4], we need only to study samples with mixture $0 \%$, $50 \%, 100 \%$ of the polymer with deuterated and undeuterated parts for both species (PMA,PMS).

In the PMA-CN study with deuterated $(100 \%)$ and non deuterated $(0 \%)$ samples the 001 bragg peak partially bilayered $S_{A}$ phase.

Because of the large temperature interval of the $S_{A}$ phase (about $42^{\circ} \mathrm{C}$ ), the temperature dependence of the characteristic sizes $R_{/ /}$and $R_{\perp}$ of the PMA-CN polymer can be studied. We report here these thermal variations (Fig.1). A very large variation of $R_{/ /}$is clearly observed. If one refers to the "Layer hopping" model of Renz and Warner [6] for the smectic phase : $R_{/ /}=L \exp \left(-E /\left(k_{\mathrm{B}} T\right)\right)$ also used in reference (7a), assuming the validity of this law, we find an activation 
energy of about $0.4 \mathrm{eV}\left(40 \mathrm{~kJ} . \mathrm{mole}^{-1}\right)$. This energy is ten times greater than that estimated for the energy of a smectic defect by Kunchenko and Svetogorsky [7a]. On the other hand this values is two or three times smaller than the values found for the self diffusion of small molecules from one smectic layer to another [10]. One striking feature is the swelling of the chain in the glassy state. Indeed as shown in figure $1, R_{\perp}$ increases in this phase while $R_{/ /}$does not change very much. This implies that there is an ordering of the polymer backbones, in agreement with the observation by DSC of a small crystallized part in that phase.

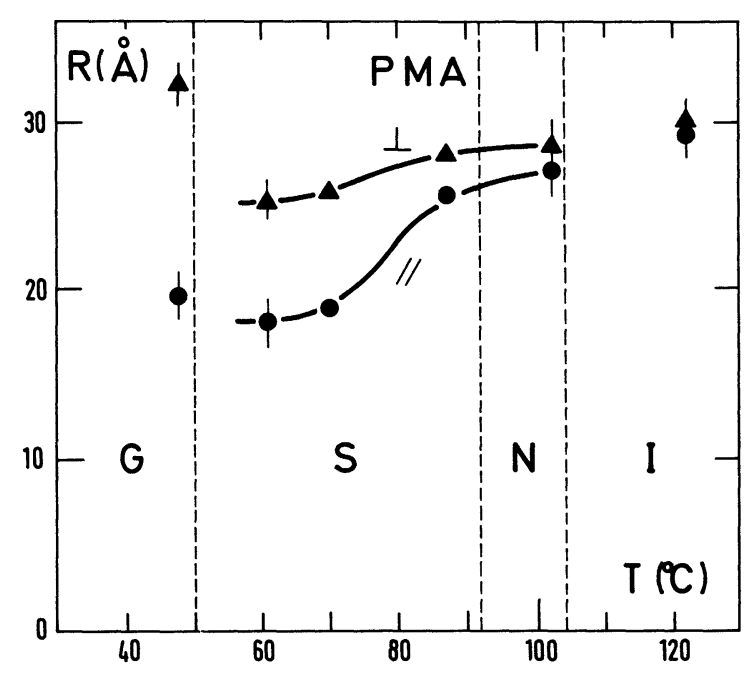

Fig.1.-Values of $R_{/ /}(\bullet)$ and $R_{\perp}(\Delta)$ versus temperature for PMA-CN. The letters G,S,N,I correspond to the liquid crystal phases (see Tab. I). The full lines are eye guide.

The substituted polymethylsiloxane PMS has been studied following the same procedure used for the PMA-CN. The neutron diffraction measurements give the same layer spacing $(\simeq 36 \AA)$ as the $\mathrm{X}$-ray scattering [8]. This is well interpreted by a partial overlapping of the mesogenic cores, although terminally non-polar [8]. Like the polymethacrylates, in the $S_{A}$ phase a third order Bragg peak from the layers is visible for the deuterated $\mathrm{PMS}-\mathrm{OCD}_{3}$ while the second order is only detected by X-ray scattering[11]. Locally, unlike the polymethacrylates the diffuse scattering spots corresponding to $\mathrm{S}_{\mathrm{A}}$ cybotactic-groups are well measured in the nematic phase. No diffuse spot at small angles is revealed in this other example of partially bilayered $S_{A}$ phase. The treatment of SANS data is also made with the formalism of equation (1) and (2). The most striking result is that $R_{/ /}$is larger than $R_{\perp}$, in both nematic and smectic phases. i.e. the anisotropy of the polymer is opposite to that of the PMA cases. It can be noted (see Fig.2) that the two slopes in the Zimm representation of equation (1) (plot of the in- verse of the intensity versus $q^{2}$ ) are not so different, with $R_{/ /}>R_{\perp}$. As it can be observed in figure 3 there is nearly no variation of these values with temperature. Surprisingly the anisotropy ratio $R_{\perp} / R_{/ /}$is quite the same in both nematic and smectic phases, this may be related to the existence of $S_{A}$ cybotactic group which are clearly observed in the nematic phase. It is of interest to note that this value $R_{\perp} / R_{/ /} \approx 0.75$ is very close to that of 0.73 found by Mattoussi et al [5] in a nematic solution of a similar polysiloxane diluted in a small nematogen solvent.

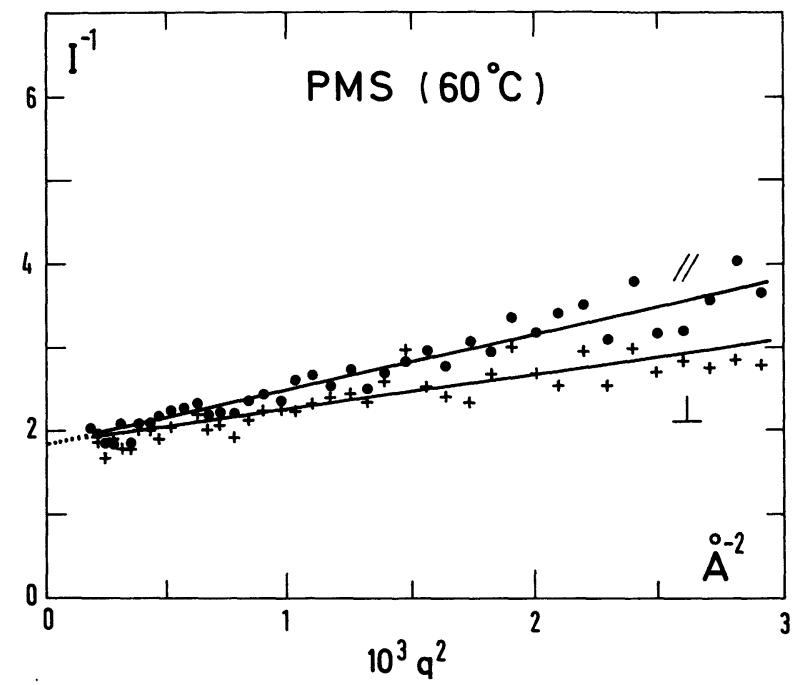

Fig.2.-Inverse of the SANS intensity $I$ plotted in arbitrary units as a function of $q^{2}$ for the PMS $-\mathrm{OCH}_{3}$ in the smectic A phase. For both direction : (•) for $q / /$, $(+)$ for $q_{\perp}$, the straight lines are obtained from a least square fit method.

Thus a first interpretation of the data can be the following : the polysiloxane backbone has a tendency to stretch along the nematic field and to keep this conformation in the smectic phase too. This can be explained by a bond $-\mathrm{O}-\left[\mathrm{Si}-\mathrm{CH}_{3}\right]-\mathrm{O}-$ less rigid than the bond $\mathrm{C}-\left[\left(\mathrm{CH}_{3}\right) \mathrm{C}\left(\mathrm{CO}_{2}\right)\right]-\mathrm{C}$, which allows the mesogenic groups to have an acute angle with respect to the backbone. Nevertheless, a totally different interpretation of the neutron results can be given because these are the tail groups which are labelled : since the molecular weight of the polymer is small, even if the backbone is confined in one smectic layer its size within the layer is smaller than the dimension of the two aligned-mesogenic molecules along the field direction (parallel direction). With such a description the value of $R_{/ /}$would correspond to the distance between two labelled terminal groups separated by two lengths of a mesogenic molecule while $R_{\perp}$ would correspond to the dimension of the backbone in a layer. If this last interpretation is correct the anisotropy will reverse as the molecular weight of the polymer increases. 


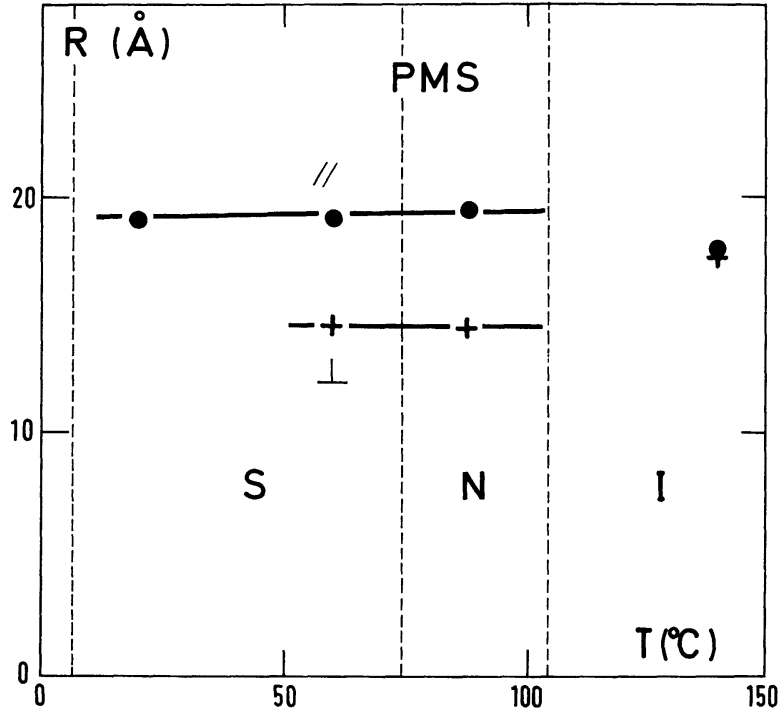

Fig.3.-Values of $R_{/ /}(\bullet)$ and $R_{\perp}(+)$ versus the temperature $T$ for the polysiloxane PMS $-\mathrm{CH}_{3}$. At $T=20^{\circ} \mathrm{C}$ $R_{/ /}$value is only reported (see text and Fig.4).

Another interesting feature of the PMS results is the aspect of the SANS spectrum at low temperature $T=20^{\circ} \mathrm{C}$. The scattered intensity $I_{/ /}$follows equation (1) but $I_{\perp}$ cannot be fitted by equation (2). This is shown clearly in figure 4 where the Zimm plot is a straight line for $q=q / /$ with $R_{/ /}=19 \AA$ but deviates strongly for $q=q_{\perp}$. Why the scattered intensity, at this temperature, present such different behaviors with the direction is an open question. By using the usual procedure of small angle technique the data registered in the perpendicular direction have been plotted in a log-log representation in order to specify the intensity variation. A good fit of the data (see Fig.4) is obtained with the following law :

$$
I(q)=I_{o} q_{\perp}^{-0.70 \pm 0.2} ; q>10^{-2} \AA^{-1} .
$$

As the scattering law for a rigid rod (length $L$ ) with a random orientation in a two dimensional space is [12] $q^{-1}$ (for $q_{\perp} L>>1$ ), this result could correspond to a stretching of the polymer in this direction. But this last interpretation has to be confirmed since the apparent molecular weight $(\sim I(\mathrm{O}))$ in the perpendicular direction seems to be far greater than that observed in the parallel one.

As a matter of conclusion, SANS by partially labelled LC polymers shows that there is not a single main direction of the anisotropy of the polymer conformation. The backbones of polymethacrylates, PMA$\mathrm{CN}$ and $\mathrm{PMA}-\mathrm{OC}_{4} \mathrm{H}_{9}$ are confined [4] in smectic layers while, with labelled terminal groups, the polysiloxanes seem to prefer the perpendicular direction (parallel to the director).

However we cannot exclude an artifact due at the same time to the low molecular weight of PMS and to the

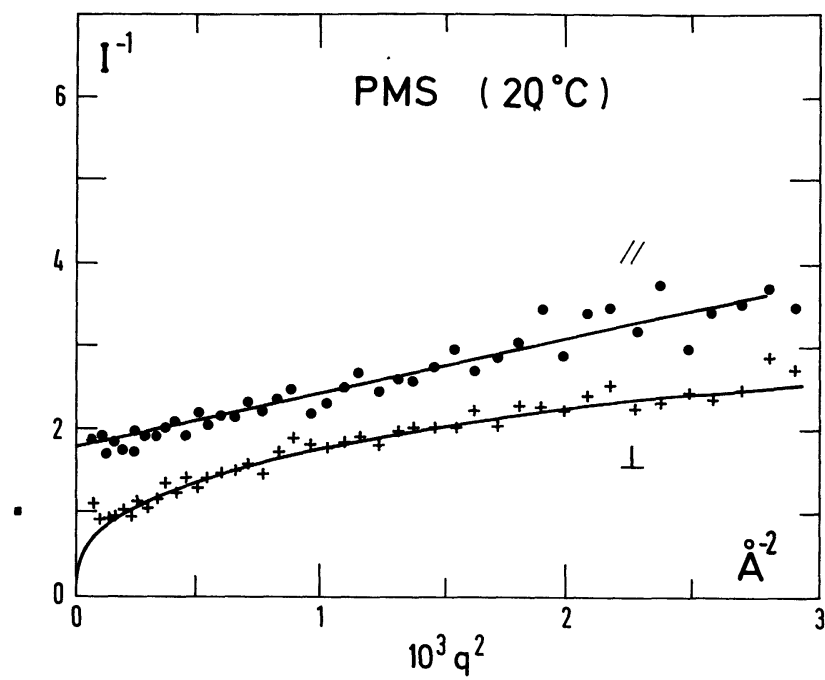

Fig.4.-Inverse of intensity $I$ plotted as a function of $q^{2}$ for PMS $-\mathrm{OCH}_{3}$. Comparison of figures 2 and 4 (the scales are identical) shows that the similarity obtained in the parallel direction $(\bullet)$ is not found in the perpendicular one $(+)$. The curved line is also a fit.

location of deuterium : this could explain the temperature independence of $R_{/ /}$. Only further measurements on polymers with larger molecular weight will allow us to conclude.

The answer is of interest since the tentative description of the structure [4] of PMA is based on semilocal segregations of the chemical compounds of a polymer. There are regions occupied by tails only, other by backbones and other ones by mesogenic cores. Thus it is important to know if this picture and the following theoretical models $[6,7]$ can be generalized to different species of LC polymers.

Thanks are due to M.F. Achard, A. Brulet, B. Farnoux for helpful assistance and useful discussions and to F. Gibert for technical assistance. We are indebted to G. Jannink for comments given on proofs from reference [12] prior to publication and to S. Shapiro for constructive remarks on the manuscript.

\section{References}

[1] (a) Polymer Liquid Crystals, CIFferI A., KRIGBAUM W.R. and MEYER R.B., (Eds) (Academic Press, N.Y.) 1982.(b) Recent Advances in Liquid Crystalline Polymers CHAPOY L.L. (Eds)(Elsevier, N.Y.) 1985.

[2] Davidson P., Keller P. and Levelut A.M., J. Physique 46 (1985) 939. 
[3] Kirste R.G., OHM H.G., Makromol. Chem. Rapid Commun. 6 (1985) 179.

[4] Keller P. et al., J. Physique Lett. 46 (1985) L-1065.

[5] MatToussi H. et al., Europhys. Lett. 2 (1986) 233.

[6] Renz W. and Warner M., Phys. Rev. Lett. 56 (1986) 1268.

[7] Kunchenko A.B. and Svetogorsky D.A., (a) J. Physique 47 (1986) 137 ; (b) Submitted to Liquid Crystals.
[8] Mauzac M. et al., Eur. Polym. J. 22 (1986) 137.

[9] Cotton J.P. et al., Macromolecules 7 (1974) 863.

[10] Kruger G.J., Phys. Rep. 82 (1982) 229.

[11] Davidson P., Private Communication.

[12] des Cloizeaux J. and Jannink G., Polymères en Solution, leur modélisation et leur structure (Editions de Physique, Les Ulis) Annexe E, Chap.7 (in press). 